\title{
Conservative Management of Bilateral Tendoachilles (TA) Rupture-A Case Report
}

\author{
Rahul Kakkar, Simon Chambers, Malcolm M. Scott \\ North Tyneside General Hospital, Rake lane, North Shields, NE29 8NH \\ E-mail: rahulkaks@rediffmail.com \\ Received March 11, 2011; revised April 27, 2011; accepted May 5, 2011
}

\begin{abstract}
Tendo Achilles ruptures are generally traumatic in origin and while bilateral tendo Achilles ruptures are a rare occurrence, most of them are associated with risk factors or pre-existing disease and generally involve trauma or sporting activities. Most of the cases of bilateral rupture are generally treated operatively. A spontaneous onset case of bilateral tendo achilles rupture is reported in a healthy man and its conservative (non operative) management discussed with a review of the literature.
\end{abstract}

Keywords: Bilateral Tendoachilles Rupture, Non-Traumatic, Spontaneous, Conservative Treatment, Cast Management

\section{Introduction}

The Achilles tendon is the thickest and strongest tendon in the body [1,2] and although forces up to 12 times body weight can be tolerated by it, it still accounts for $20 \%$ of all large tendon ruptures [2]. Trauma and sporting activities have been implicated as the most common cause of tendoachilles rupture $[3,4]$ with the injury mechanism involving sudden and powerful contraction of the gastrocnemius and soleus muscles on a dorsiflexed foot and usually affecting men aged 30 - 50 years [5].

But, spontaneous bilateral Achilles tendon ruptures are a rare occurrence [1,6-12] and account for about $1 \%$ of all Achilles tendon ruptures [9,11]. Risk factors for these type of injuries include corticosteroid use [12], limb ischemia [13], anabolic steroids [14], fluoroquinolones [15], chronic pain, previous Achilles tendon rupture [16] and rheumatoid arthritis, SLE [17] .To our knowledge, only a few reports on spontaneous bilateral Achilles tendon tears have been published, most with associated risk factors but with satisfactory outcomes and there is only one other case report of a bilateral rupture of the tendo Achilles without any associated risk factors [18]. We present a case of spontaneous bilateral tendoachilles rupture in an otherwise healthy 49 year old man which was successfully treated conservatively with two different management protocols.

\section{Case Report}

A 49 year-old man attended fracture clinic with a right TA rupture which was confirmed clinically. He had been on holiday and was walking near a waterfall when he felt pain in his right tendo Achilles area and was unable to walk after that. He had no obvious risk factors for tendon rupture; he was a non-smoker, with no recent or past history of steroid or fluoroquinolone use. He had no metabolic derangements, and blood tests (FBC, U+E, LFT, TFT,cholesterol, rheumatoid factor and urate) were all within normal limits. Following a discussion about the relative merits and risks of operative versus non-operative treatment, he opted for non-operative treatment. He had an above knee equinus cast applied with knee flexed about 20 degrees and was asked to be non weight bearing on the right leg.

Unfortunately he re-attended casualty 10 days into his course of non-operative treatment complaining of left TA rupture. He was going upstairs using his crutches, when he felt his left TA rupture, which he described as a 'tearing' sensation. On examination he had bruising; swelling and tenderness of the left TA with a palpable gap present approximately $4 \mathrm{~cm}$ proximal to its insertion into os calcis. The calf squeeze test was positive, i.e. no ankle plantar flexion was present on squeezing the calf. He was placed into a short-leg (below knee) equinus cast and admitted to the ward while arrangements were put in 
place to allow his safe discharge. We treated both sides differently with the right leg in above knee equinus cast and the other in below knee equines cast (Figure 1) for first three weeks in an effort to provide him some mobility as putting both legs in Above Knee casts would be cumbersome for him. Happily his brother had an adapted home due to being a wheelchair user himself, and so our patient was able to be discharged fairly promptly despite being non-weight-bearing with bilateral-leg casts. This initial equinus cast was followed by a below knee midequinus for three weeks and then a walking plantigrade cast for three weeks (total time in casts was 9 weeks for each leg). Both sides healed well and there was no apparent difference in the two sides and healing times were similar.

\section{Discussion}

Bilateral TendoAchilles rupture is rare, but is documented in the medical literature. In 2004 Hayes, et al. [19] reviewed all of the published cases of bilateral rupture and found that of 26 cases, 13 were due to exogenous steroid treatment, three were due to significant trauma, three occurred in renal transplant patients, two were attributable to antibiotics of the fluoroquinilone family, one was due to endogenous corticosteroid excess

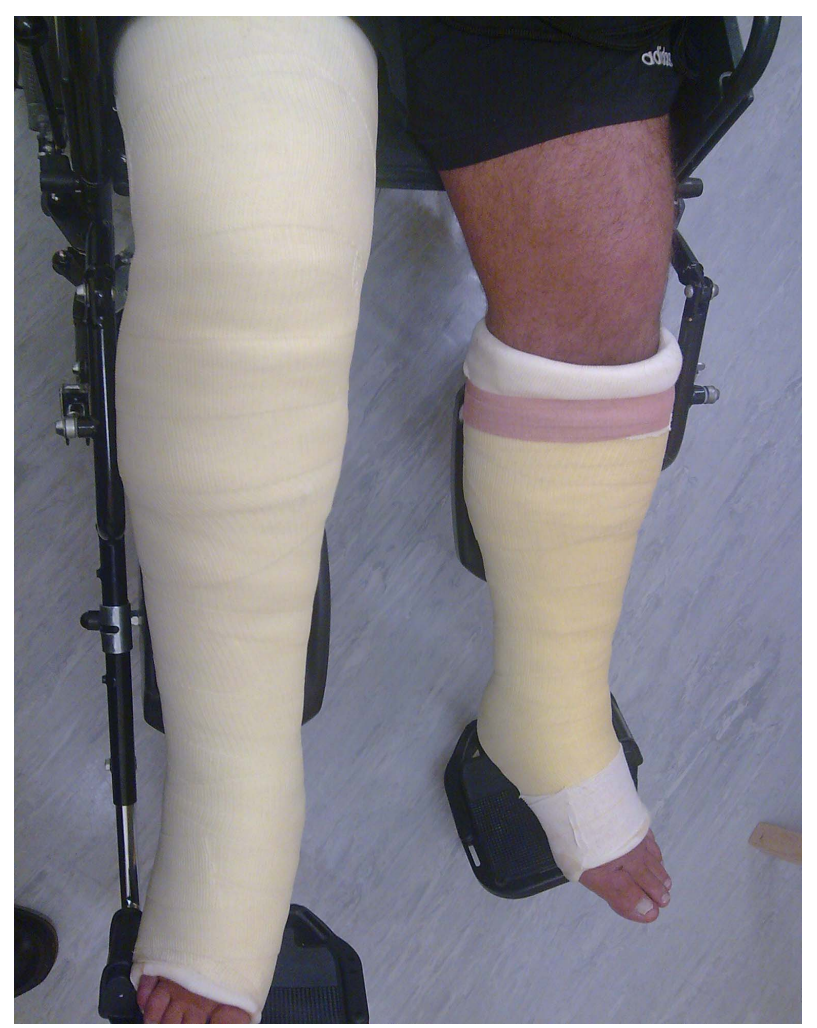

Figure 1. Clinical picture showing above knee cast on right side and below knee cast on the left.
(Cushing's), one due to limb ischaemia, one diabetes, one systemic lupus. Only one report had no cause identified, [20] but there was no other information about the medical background other than that she was not on steroids. Hayes, et al. [19] presented their case and suggested that smoking may have been a causative factor. Rao, et al. [21] reported another case but their patient had hypothyroidism and was taking thyroxine and they surmised that hypercholesterolemia and hypothyroidism lead to degenerate tendon which eventually ruptured. Habusta [9] reported two cases in healthy patients however both patients had long term Achilles tendonitis and were gymnasts. Specific bilateral forces generated through the Achilles tendons of experienced older gymnasts predispose them to chronic bilateral wear and acute bilateral simultaneous ruptures during specific manoeuvres. The only other case report in the literature without any causative factor is by Garg, et al. [18] and their patient was a healthy woman but they treated her operatively.

Conservative treatment of Achilles tendon tears has been considered a reasonable form of treatment. It has a relatively good outcome and the risk of wound breakdown is eliminated. In a retrospective cohort study published in 2003, Weber, et al. [22], found that non-operative treatment with an equinus ankle cast and boot for 12 weeks was as effective as surgical treatment in return to sports and ultimate strength as operative treatment. Moreover, they also reported that non-operative treated patients had a much faster subsidence of pain, return to unaided walking, and return to work. Eames, et al. [23], showed similar outcomes between surgical and non-surgical treatment but closed treatment had a lower minor complication rate.

Sekiya, et al. [24] and Wray, et al. [25] have demonstrated in cadaveric studies that knee flexion position does not influence the separation of the tendo Achilles ends while the plantarflexion position of the ankle does significantly affect the separation of the tendoachilles ends. Wray, et al. [25] also showed that the ankle needs to be in at least 28 degrees of plantarflexion for the two ruptured ends of the tendoachilles to meet. Therefore the only factor to be controlled is the ankle position, which can be controlled in a below knee cast and an above knee cast is not necessary. There is only one other report in the literature where the patient was treated conservatively for bilateral tendo Achilles ruptures [26]. Their patient had severe COPD and had been treated with steroids. Bilateral cast brace treatment was provided for this patient considering the poor general health. Unfortunately, their patients' right tendo-achilles did not heal with brace management perhaps as result of him being on steroids. We think that our patient achieved satisfac- 
tory healing as he was not on steroids and did not have any other risk factors and thus, possibly had healthier tendons to start with which healed without any consequence.

Our case presents a unique opportunity to examine conservative treatment methods in a healthy patient. Although different management was used (above knee versus below knee casts) for each side, the outcomes on both sides were similar and in this case it did not make a difference whether an above knee or a below knee cast was used for the initial treatment as the tendons healed on both the sides and the patient returned to their pre-injury activity levels. This also demonstrates that the amount of knee extension/flexion has no bearing on the tendoachilles healing and that the only factor affecting the separation of tendoachilles ends and thus their healing is the plantarflexion of the ankle.

This case is only the second reported case of bilateral rupture in a healthy patient and our case demonstrates that bilateral tendoachilles rupture can be treated conservatively with a good outcome.

\section{References}

[1] A. Mehra, R. Case and C. Croucher, "Bilateral Simultaneous Spontaneous Rupture of the Achilles Tendon," Hospital Medicine, Vol. 65, No. 5, 2004, pp. 308-309.

[2] N. Maffulli, M. G. Kenward, V. Testa, G. Capasso, R. Regine and J. B. King, "Clinical Diagnosis of Achilles Tendinopathy with Tendinosis," Clinical Journal of Sport Medicine, Vol. 13, No. 1, 2003, pp. 11-15. doi:10.1097/00042752-200301000-00003

[3] M. Paavola, P. Kannus, T. Paakkala, M. Pasanen and M. Jarvinen, "Long-Term Prognosis of Patients with Achilles Tendinopathy. An Observational 8-Year Follow-up Study," American Journal of Sports Medicine, Vol. 28, No. 5, 2000, pp. 634-642.

[4] M. Paavola, S. Orava, J. Leppilahti, P. Kannus and M. Jarvinen, "Chronic Achilles Tendon Overuse Injury: Complications after Surgical Treatment. An Analysis of 432 Consecutive Patients," American Journal of Sports Medicine, Vol. 28, No. 1, 2000, pp. 77-82.

[5] L. Jozsa, M. Kvist, B. J. Balint, et al., "The Role of Recreational Sport Activity in Achilles Tendon Rupture. A Clinical, Pathoanatomical, and Sociological Study of 292 Cases," American Journal of Sports Medicine, Vol. 17, No. 3, 1989, pp. 338-343. doi:10.1177/036354658901700305

[6] D. P. Hanlon, "Bilateral Achilles Tendon Rupture: An Unusual Occurrence,” Journal of Emergency Medicine, Vol. 10, No. 5, 1992, pp. 559-560. doi:10.1016/0736-4679(92)90136-H

[7] D. R. Baruah, "Spontaneous Rupture of Bilateral Achilles Tendon of a Patient on Long-Term Systemic Steroid Therapy,” Unfallheilkunde, Vol. 87, No. 1, 1984, pp. 35-36.

[8] W. Dickey and V. Patterson, "Bilateral Achilles Tendon
Rupture Simulating Peripheral Neuropathy: Unusual Complication of Steroid Therapy," Journal of the Royal Society of Medicine, Vol. 80, 1987, pp. 386-387.

[9] S. F. Habusta, "Bilateral Simultaneous Rupture of the Achilles Tendon. A Rare Traumatic Injury,” Clinical Orthopaedics and Related Research, Vol. 320, 1995, pp. 231-234.

[10] J. F. Haines, "Bilateral Rupture of the Achilles Tendon in Patients on Steroid Therapy," Annals of the Rheumatic Diseases, Vol. 42, No. 6, 1983, pp. 652-654. doi:10.1136/ard.42.6.652

[11] M. Kelly, M. Dodds, J. S. Huntley and C. M. Robinson, "Bilateral Concurrent Rupture of the Achilles Tendon in the Absence of Risk Factors,” Hospital Medicine, Vol. 65, 2004, pp. 310-311.

[12] S. Orava, M. Hurme and J. Leppilahti, "Bilateral Achilles Tendon Rupture: A Report on Two Cases,” Scandinavian Journal of Medicine and Science in Sports, Vol. 6, No. 5, 1996, pp. 309-312. doi:10.1111/j.1600-0838.1996.tb00476.x

[13] D. D. Shukla, "Bilateral Spontaneous Rupture of Achilles Tendon Secondary to Limb Ischemia: A Case Report," Journal of Foot and Ankle Surgery, Vol. 41, No. 5, 2002, pp. 328-329. doi:10.1016/S1067-2516(02)80052-7

[14] V. Battista, J. Combs and W. J. Warme, “Asynchronous Bilateral Achilles Tendon Ruptures and Androstenediol Use,” American Journal of Sports Medicine, Vol. 31, No. 6, 2003, pp. 1007-1009.

[15] W. T. Lee and J. F. Collins, "Ciprofloxacin Associated Bilateral Achilles Tendon Rupture," Australian and New Zealand Journal of Medicine, Vol. 22, No. 5, 1992, p. 500.

[16] A. Aroen, D. Helgo, O. G. Granlund and R. Bahr, "Contralateral Tendon Rupture Risk is Increased in Individuals with a Previous Achilles Tendon Rupture," Scandinavian Journal of Medicine \& Science in Sports, Vol. 14, No. 1, 2004, pp. 30-33. doi:10.1111/j.1600-0838.2004.00344.x

[17] K. Matsumoto, S. Hukuda, J. Nishioka and S. Asajima, "Rupture of the Achilles Tendon in Rheumatoid Arthritis with Histologic Evidence of Enthesitis. A Case Report," Clinical Orthopaedics and Related Research, Vol. 280, 1992, pp. 235-240.

[18] S. Garg and M. Thilagarajah, "Bilateral Rupture of Achilles Tendon (bilrat) without Predisposing Systemic Disease or Steroid Use: A Case Report and Review of the Literature," The Internet Journal of Orthopedic Surgery. Vol. 13, No. 2, 2009.

[19] T. Hayes, D. McClelland and N. Mafulli, "Metasynchronous Bilateral Achilles Tendon Rupture. Bulletin,” Hospital for Joint Diseases, Vol. 61, No. 3-4, pp. 2003-2004.

[20] J. H. Mayer, "Correspondence. Bilateral Rupture of Achilles Tendons,” British Medical Journal, Vol. 1, 1961, p. 1830. doi:10.1136/bmj.1.5242.1830

[21] S. K. Rao, B. C. Navadgi and A. Vasdev, "Bilateral Spontaneous Rupture of Achilles Tendons: A Case Report," Journal of Orthopaedic Surgery, Vol. 13, No. 2, 2005, 178 $-180$

[22] M. Weber, M. Niemann, R. Lanz and T. Muller, "Non- 
operative Treatment of Acute Rupture of the Achilles Tendon: Results of a New Protocol and Comparison with Operative Rreatment," American Journal of Sports Medicine, Vol. 31, 2003, pp. 685-691.

[23] M. H. Eames, N.W. Eames, K. R. McCarthy and R. G. Wallace, "An Audit of the Combined Non-Operative and Orthotic Management of Ruptured Tendo Achillis,” Injury, Vol. 28, No. 4, 1997, pp. 289-292.

[24] J. K. Sekiya, K. E. Evensen, P. J. L. Jebson and J. E. Kuhn, "The Effect of Knee and Ankle Position on Displacement of Achilles Tendon Ruptures in a Cadaveric Model. Implications for Nonoperative Management,”
American Journal of Sports Medicine, Vol. 27, No. 5, 1999, pp. 632-635.

[25] W. H. Wray, C. Regan, S. Patel, R. May and S. Parekh, "Positional Effects of the Knee and Ankle on the Ends of Acute Achilles Tendon Ruptures,” Foot \& Ankle Specialist, Vol. 2, No. 5, 2009, pp. 214-218. doi:10.1177/1938640009346449

[26] M. A. Ramirez and L. C. Richardson, "Pulmonary Embolism Associated with Spontaneous Bilateral Achilles Tendon Rupture," Journal of Foot and Ankle Surgery, Vol. 46, No. 4, 2007, pp. 283-287. doi:10.1053/j.jfas.2007.03.014 